Original Research Paper

\title{
Lentinan Properties in Anticancer Therapy: A Review on the Last 12-Year Literature
}

\author{
${ }^{1}$ Luca Vannucci, ${ }^{1}$ Petr Š́ma, ${ }^{2}$ Vaclav Vetvicka and ${ }^{1}$ Jiří Křrižan \\ ${ }^{1}$ Laboratory of Immunotherapy, Institute of Microbiology v.v.i., Czech Academy of Sciences, Prague, Czech Republic \\ ${ }^{2}$ Department of Pathology, School of Medicine, University of Louisville, USA
}

\author{
Article history \\ Received: 15-11-2016 \\ Revised: 19-01-2017 \\ Accepted: 29-03-2017 \\ Corresponding Author: \\ Luca Vannucci \\ Laboratory of Immunotherapy, \\ Institute of Microbiology v.v.i., \\ Czech Academy of Sciences, \\ Prague, Czech Republic \\ Tell: +420241062394 \\ Email: vannucci@biomed.cas.cz
}

\begin{abstract}
The effects of $\beta$-glucan in cancer are the most studied biological effects of $\beta$-glucan. From all available $\beta$-glucans, the most data are available on lentinan, mostly because it is used in clinical practice for over 20 years. Current review represent a state-of-the-art comprehensive review of knowledge obtained in last twelve years.
\end{abstract}

Keywords: Lentinan, Glucan, Cancer, Immunity

\section{Introduction}

In recent years, the global market of natural products, especially medicinal herbs and mushroom, has had an intensive growth. Even though the majority of these products are sold as dietary supplements to increase the health and wellness of the individuals, many are presented as beneficial "drugs" and used in the traditional medicine for curing many illnesses including cancer (e.g., aloe vera, red beetroot juice, mushrooms) (Parveen et al., 2015).

Mushrooms, used for centuries in the Eastern traditional medicine, represent a main source of one of the most attractive and more studied class of plant derivatives, the glucans. Glucans are extracted from various sources (from yeast to edible mushrooms) and are D-glucose-based polysaccharides. They can be $\alpha$-Dglucans, $\beta$-D-glucans, or mixed $\alpha, \beta$-D-glucans due to their glucose anomeric structure, with effects on their activity (Vannucci et al., 2013). Repeated $\beta$-D-glucose units joined together in linear chains by $\beta$-bonds constitute the basic $\beta$-glucan structure, which can extend either from carbon 1 of one saccharide ring to carbon 3 of the next $(\beta 1 \rightarrow 3)$ or to carbon $4(\beta 1 \rightarrow 4)$, or to carbon $6(\beta 1 \rightarrow 6)$ (Fig. 1) (Tzianabos, 2000).

Both $(1 \rightarrow 3)$ - and $(1 \rightarrow 6)$ - $\beta$-glucans (e.g., lentinan and pleuran, from Lentinusedodes and Pleurotus ostreatus, respectively) are based on different types of glycosidic bonds and are considered medically useful, as established by their use in traditional Asiatic medicine and by results of modern scientific and medical studies (Molitoris, 1994; Novak and Vetvicka, 2009).

Of the various types of $\beta$-glucans, lentinan has recently become the more popular as an active immune modulator with anticancer properties. Frequently proposed by commercial sites as dietary supplements (https://www.drugs.com/npp/lentinan.html), information about the properties of lentinan can also be found on some cancer institute websites (https://www.mskcc.org/cancer-care/integrativemedicine/herbs/lentinan).

\section{Lentinan}

Lentinan is a $\beta-(1 \rightarrow 6)$ branched $\beta-(1 \rightarrow 3)$-glucan extracted from the mushroom Lentinus edodes also known as shiitake (Fig. 2). Lentinan immunomodulatory properties are known for a long time. They include macrophage activation, Dendritic Cell (DC) activation by Dectin-1 binding, increase of cytotoxic $\mathrm{T}$ lymphocytes and Natural Killer (NK) activity and enhancement of Th1 Vs Th2 response (Chihara, 1983; Chihara et al., 1987; Goodridge et al., 2011; Leibundgut-Landmann et al., 2008).

Various studies have shown lentinan binding to monocytes and to neutrophils and their activation supports the maturation of lymphoid cells. Dectin-1 as well as complement factors appear to be targeted by 
lentinan. Competition experiments for the complement receptors CR1 and CR3 demonstrated inhibition of lentinan binding to monocytes (Oka et al., 1996; Xia et al., 1999). The beneficial effect in anticancer therapy of the association between lentinan and monoclonal antibodies (e.g., trastuzumab) was explained by their synergistic effect on the activation of complement system (antibodydependent cellular cytotoxicity and the complementdependent cytotoxicity) (Ina et al., 2013).

Direct effects on cancer cells are also reported for lentinan, like downregulation of TERT and $M Y C$ expression, apoptosis and inhibition of cell proliferation secondary to enhanced tumor suppressor TP53 expression, cell cycle arresting p21 and pro-apoptotic proteins of $B A X$ and $C A S P 3 / 9$. Downregulation of $P A R P 1$ and anti-apoptotic protein $B C L 2$ expressions were also found in the treated tumor tissues.

These and others previous biological and immunological investigations (Wasser, 2002) strongly suggest the possibility that a supplementation with lentinan might exert positive effects even on cancer patients. To better understand the perspective role of lentinan in anticancer treatments, we have reviewed and summarized the literature of the last 12 years, according to PubMed, reporting data from in vitro and in vivo tests as well as clinical studies in which lentinan effects were evaluated in relation to chemotherapy.

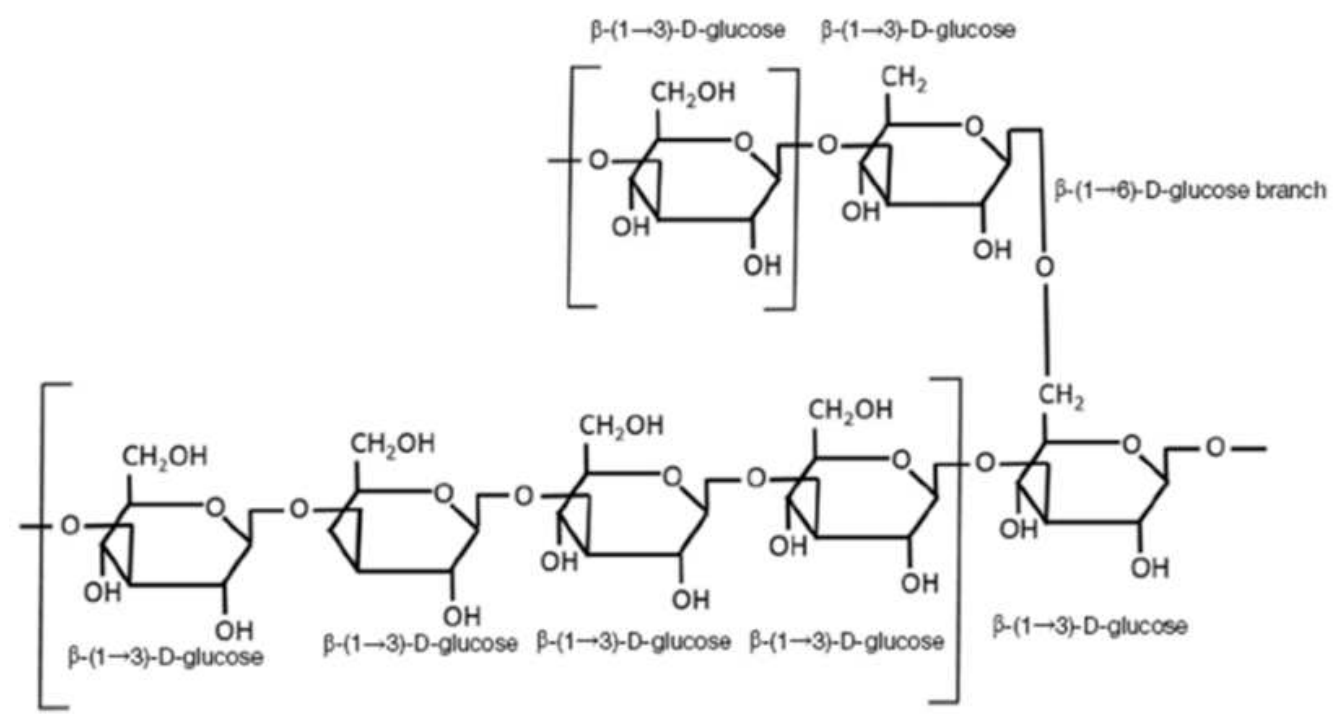

Fig. 1. $\beta$-D-glucan structure. Bonds $(1 \rightarrow 3)$ - and $(1 \rightarrow 6)$ - are shown

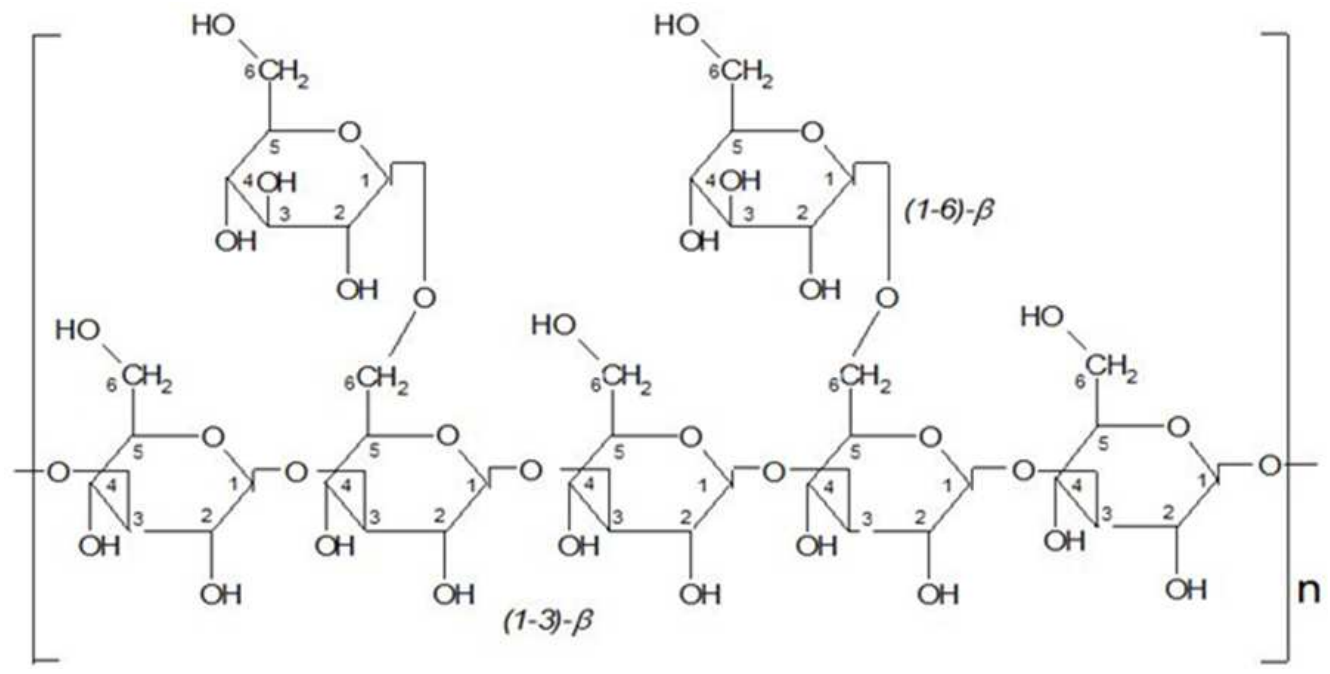

Fig. 2. Lentinan chain structure 


\section{Published Papers and Type of Cancers}

From January 2005 through August 2016, we retrieved a list of 39 papers about lentinan in the treatment of various types of cancers from PubMed (Table 1). Two of these papers reported the results of a meta-analysis on 650 individual cases of advanced gastric cancer from five trials (Oba et al., 2009) and a collection of observations on groups of patients with various gastrointestinal cancers (Yagi et al., 2010). In all cases, a comparison was done between patients who were treated with lentinan associated to chemotherapy versus patients treated with chemotherapy only. Single patient case reports and papers in the original language with insufficient data reported in the English language were excluded from our review. This limited the full evaluation of all studies, however a sufficiently detailed English summary of the design of the study and of the most relevant results was available in most papers from international journals we reviewed.

The most studied cancer was gastric cancer (with 8 studies and a meta-analysis of clinical results) representing approximately one-fourth of all literature (23.1\%) (Oba et al., 2009). Sarcoma, the second most studied, was evaluated only in experimental conditions, either in vitro or in vivo, in animal models (15.4\%). Colon and lung (Non-Small Cell Carcinoma [NSCLC]) cancers followed, $12.8 \%$ and $10.3 \%$, respectively, with both experimental and clinical studies (Fig. 3).

The total bulk of papers was almost equally subdivided between experimental (in vitro or in vivo) and clinical studies (Table 2). The studies about effect of lentinan in the treatment of gastrointestinal cancers were prevalent (19 papers) and, within the clinical studies, the advanced gastric cancer was the most investigated. Within the experimental studies, the sarcoma model was the most used (Table 3).

A total of 1753 patients were evaluated for response to lentinan in various advanced cancer conditions, with the largest number in the lung cancer group, followed by the gastric cancer patients (Table 4). To this number we added the 650 gastric cancer patients reported in Oba's paper (Oba et al., 2009) and another 72 patients evaluated by Yagi et al. (2010).

Table 1. Publications for each type of lentinan-tested cancer on PubMed 2005-2016

\begin{tabular}{lll}
\hline Cancer & n. papers & $\%$ \\
\hline Gastric & 9 & 23,08 \\
Sarcoma & 6 & 15,39 \\
Colon & 5 & 12,82 \\
Lung & 4 & 10,26 \\
Liver & 4 & 10,26 \\
Bladder & 2 & 5,13 \\
Head and neck & 2 & 5,13 \\
Endocavitary effusions & 2 & 5,13 \\
Oesophagus & 1 & 2,56 \\
Pancreas & 1 & 2,56 \\
Acute myelod leukemia & 1 & 2,56 \\
Melanoma & 1 & 2,56 \\
Various cancers & 1 & 2,56 \\
Total of publications & 39 & 100 \\
WoS publications 2005-2016 & & \\
\hline
\end{tabular}

Table 2. Proportion of publications about experimental Vs clinical studies

\begin{tabular}{lll}
\hline & n. papers & $\%$ \\
\hline Experimental & 20 & 51.28 \\
Clinical & 19 & 48.72 \\
Total & 39 & 100 \\
\hline
\end{tabular}

Table 3. Experimental and clinical papers for each type of cancer tested with lentinan

\begin{tabular}{llll}
\hline Cancer & n. papers & Experimental & Clinical \\
\hline Gastric & 9 & 1 & 8 \\
Sarcoma & 6 & 6 & 0 \\
Colon & 5 & 4 & 1 \\
Lung & 4 & 1 & 3 \\
Liver & 4 & 2 & 2 \\
Bladder & 2 & 2 & 0 \\
Oesophagus & 1 & 0 & 1 \\
Head and neck & 2 & 2 & 0 \\
Malignant effusions & 2 & 0 & 2 \\
Pancreas & 1 & 0 & 1 \\
Acute myelod leukemia & 1 & 1 & 0 \\
Melanoma & 1 & 1 & 0 \\
Various cancers & 1 & 0 & 1 \\
Total of publications & 39 & 20 & 19 \\
\hline
\end{tabular}


Table 4. Number of patients studied for each type of cancer

\begin{tabular}{lll}
\hline Cancer & Clinical studies & patients/study * \\
\hline Oesophagus & 1 & 50 \\
Gastric & 7 & 359 \\
Colon & 1 & 80 \\
Liver & 2 & 114 \\
Pancreas & 1 & 29 \\
Lung & 3 & 1091 \\
Malignant effusions & 2 & 30 \\
Total * & 17 & 1753 \\
\hline
\end{tabular}

*Includes data previously evaluated and reported (Oba et al., 2009; Yagi et al., 2010)

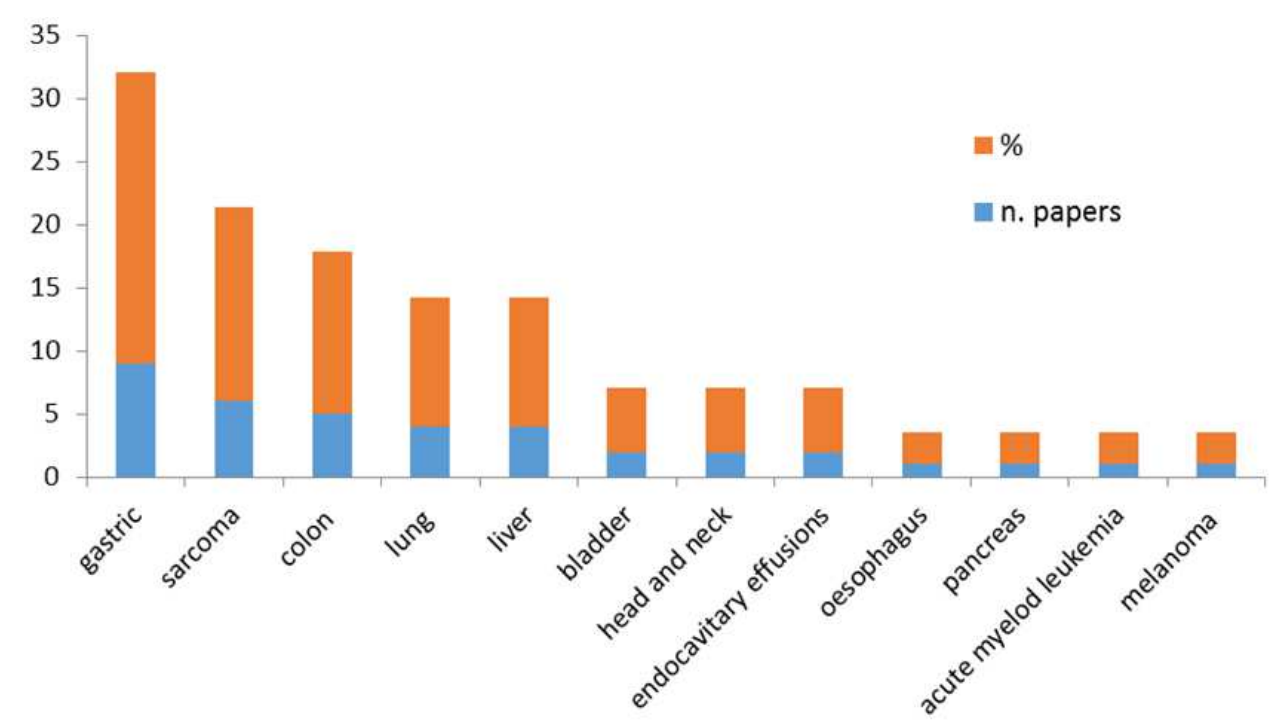

Fig. 3. Publications on lentinan according to the various types of cancers

\section{Gastric Cancer}

The general incidence of gastric cancer is on the decline; nevertheless, the incidence and mortality rate remains high, particularly in the Asian and Eastern Europe populations. The 5-year-survival rate from diagnosis, despite the various protocols of chemotherapy, remains less than $30 \%$ (Ferlay et al., 2015). An experimental study of BGC823 cells in vitro, evaluated the cytotoxic effect produced by the association of lentinan to docetaxel and cisplatin. Using different concentrations, it was shown by the tetrazolium-based colorimetric assay that the viability of the cells was inversely proportional to the concentration of the administered drugs. Similarly, the inhibition level of proliferation and the induction of apoptosis evaluated by flow cytometry using the Annexin V/propidium iodide method confirmed the dose concentration-related effect. The association of lentinan to the chemotherapeutic drugs produced enhancement of the anticancer effects: At a lower dosage, the docetaxel and cisplatin in association with lentinan was more effective than the two drugs alone at higher dosage (Zhao et al., 2013). This positive effect has been confirmed in various clinical reports, underlining the possibility to prolong the treatments in advanced cancer conditions (in which many times, due to the reduced performance status of the patients, it is necessary to interrupt or re-modulate the therapy at lower dosages) due to an improved quality of life with consequent effects also on the total survival rate.

The seven clinical studies devoted to lentinan used in association with the chemotherapy in advanced, unresectable, or recurrent gastric cancer account for a total of 359 patients almost equally divided between chemotherapy only and lentinan + chemotherapy treated groups (Higashi et al., 2012; Hori et al., 2011; Ina et al., 2011; Kataoka et al., 2009; Nimura et al., 2006; Yoshino et al., 2016; 2010a). All studies used the chemotherapeutic drug combination S-1, alone or associated with either doxetacel or cisplatin, as the principal treatment, with or without administration of lentinan either intravenously ( $2 \mathrm{mg} /$ body in $30 \mathrm{~min}$ every 1 to 3 weeks depending on the protocol) or by daily oral administration (15 mg of a superfine dispersed lentinan/bag/day) (Yagi et al., 2010; Yoshino et al., 2016). S-1 treatment was developed in Japan and is a 
commercialized combination of the orally administered 5-fluorouracil pre-drug tegafur with gimeracil and oteracil as metabolic enhancers, associated in a 1:0.4:1 molar ratio (Shirasaka et al., 1996). It has become a standard treatment, approved in Japan for advanced gastric cancer therapy and expected to eventually be used in association with other anticancer drugs. During these treatments, the patients were followed by standard evaluation of therapy toxicity, side effects occurrence, performance status, quality of life questionnaire, time-totreatment failure, overall response rate and survival. In some studies, the evaluation of the lentinan-binding monocytes to achieve a further parameter for possible discrimination between responders and no responders to the $\beta$-glucan (Kataoka et al., 2009; Yoshino et al., 2016) and the granulocyte/lymphocyte ratio (Ina et al., 2011; Nimura et al., 2006), were also applied.

Independent from standard chemotherapeutic protocol, the addition of lentinan produced some favorable effects noted in almost all studies. These included an improvement in the quality of life, a trend in reduction of the chemotherapy side effects, sometimes not reaching a statistical significance (no differences in the intensity of side effects when occurring and in two cases constipation [grade 2] and nausea [grade 1] were in direct relationship to superfine dispersed lentinan administration) (Higashi et al., 2012; Hori et al., 2011; Kataoka et al., 2009; Nimura et al., 2006; Yagi et al., 2010; Yoshino et al., 2010a). It was the possibility to prolong chemotherapy administration, improving quality of life; and prolonging survival was also reported (Ina et al., 2011; Nimura et al., 2006; Yoshino et al., 2016). The individual patientbased meta-analysis conducted by Oba et al. (2009) concluded that the addition of lentinan to the chemotherapy treatment produced a statistically significant prolongation of survival in this group of patients Vs those in the chemotherapy-only group $(P=$ 0.011). Nimura et al. (2006) also reported longer survival time using lentinan. The recent study by Yoshino et al. (2016), however, is the only one in which the authors did not obtain positive results. Patients (154 with advanced cancer and 155 with recurrent cancer) were randomly assigned to either the chemotherapy group or the chemotherapy + lentinan group. Significant improvement of the quality of life was not found, the reduction of chemotherapy dose for side effects was $19.5 \%$ in chemotherapy + lentinan Vs $17.8 \%$ of chemotherapy-only patients and the median of chemotherapy administration was three doses for S-1 treated patients and two in the lentinan association, which, in this cases, affected negatively the time-to-treatment failure. The lentinan treatment was prolonged to a median of 22 weeks ( 1 dose IV/wk). The lentinan administration did not influenced the survival time. The authors concluded that lentinan combined with S-1 chemotherapy was not effective. Nevertheless, they reported a unique exception in a subgroup of patients with the lentinan-binding monocyte value $>2 \%$. This condition appeared favorable to prolong the overall survival: The 3-year survival rate in chemotherapy + lentinan treated patients was $24.1 \mathrm{Vs}$ $3.1 \%$ of chemotherapy-only. The lentinan-binding monocyte test was proposed as a possible prognostic indicator (Yoshino et al., 2010a). Finally, another subgroup appeared to be responsive to the lentinan: Oba et al. (2009) studied patients positive or negative for metastases in the lymph nodes and found the lymph node-positive patients had a better outcome than the lymph node-negative patients.

With the exception of Yoshino's report, all other studies indicate lentinan is a useful supplement for palliative interventions in advanced gastric cancer treatment, increasing the quality of life and tolerance to chemotherapy side effects. Some authors also underlined a positive effect of lentinan on the overall survival, secondary to more prolonged periods of chemotherapy treatment permitted by better conditions of the patients. Importantly, a significant correlation was found between better outcome and level of lentinan-binding monocytes.

\section{Colon Cancer}

In the evaluated period, five papers (four experimental and one clinical) were published and reviewed. Three experimental studies were performed in vitro, using human colorectal cancer cell lines (Jeff et al., 2013; Sreenivasulu et al., 2010; Zheng et al., 2015) and one in vivo, using a mouse syngeneic tumor model (Mushiake et al., 2005). The clinical study reported the effects of superfine dispersed lentinan administration and can be evaluated together with the results of Yagi's study (Yagi et al., 2010) in which 25 colorectal cancer patients were similarly treated (Hazama et al., 2009; Yagi et al., 2010).

The in vitro experimental studies were addressed to test new and various polysaccharides isolated from fruiting bodies of Lentinus edodes. Jeff et al. ( 2013) characterized a novel mannogalactoglucan, while Zheng et al. (2015) evaluated six water-soluble polysaccharides. In both studies, the human colon cancer cell lines HT-29 and HTC-116 were exposed to the purified lentinan fractions obtained by water extraction. These fractions were heteroglucans at various ratios of glucose (Glc), mannose (Man) and galactose (Gal) molecules linked in different positions $(1 \rightarrow 3,1 \rightarrow 6$, $1 \rightarrow 4)$. In both studies, the polysaccharides were tested with mouse sarcoma 180 (S-180) cell line. Interestingly, while the various lentinan fractions showed a certain cytotoxic effect on the cancer cells, they resulted more active against the no adherent S-180 than the adherent 
colon cancer cell lines. The antitumor effect was dose dependent (Jeff et al., 2013), with effective dose of $5 \mathrm{mg}$ $\mathrm{mL}^{-1}$ for all types of cell lines (Zheng et al., 2015). They also indicated a relationship between anticancer effect and the variation of monosaccharide content (Man and Gal), molecular weight and possible bound proteins to the fractions. A further study by Sreenivasulu et al. (2010) on the DLD-1 cancer cell line highlighted a possible role of lentinan as inhibitor of telomerase through downregulation of the $M Y C$ and $T E R T$. Using progressive concentrations from 2 to 10 $\mu \mathrm{g} \mathrm{mL} L^{-1}$ of their own lentinan preparation, the effect was dose dependent.

An in vivo test was performed by Mushiake et al. (2005). They used the mouse colonic adenocarcinoma CT26 cell line to produce solid cancers after inoculation in syngeneic Balb/c mice and in athymic mice (with innate immunity but without $\mathrm{T}$-cell immunity). The aim was to induct DC activation by lentinan. The DC activation is fundamental for antigen presentation to $\mathrm{T}$ cells eliciting the specific anticancer immune response (Schreibelt et al., 2013). The scheme of anticancer treatment was the S-1 with or without lentinan. The results indicated that the association of lentinan with the S-1 chemotherapy was beneficial. In fact, the tumors of double-treated mice were presenting more intense infiltrate of DC expressing the CD86 activation marker and in the spleen the cytotoxic $\mathrm{T}$ cells presented enhanced proliferation and cytotoxicity in comparison to S-1-only treated mice $(p<0.05)$. The importance of the collaboration between more active DCs and T cells was better shown by the results on animal survival in the Balb/c Vs the athymic mice. These T-cell-deficient mice had a significantly shorter survival than the fully competent mice $(p<0.05)$.

A new oral preparation of lentinan, the superfine dispersed lentinan, was used in a multicentric clinical study on various digestive cancers (Hazama et al., 2009). The superfine dispersed lentinan, considered to be substitutive of the parenteral administration because of its ease in penetrating the digestive mucosa with higher therapeutic levels than previous oral preparations, was administered to 80 colorectal cancer patients at the dose of $15 \mathrm{mg} /$ day for 12 weeks in association with various protocols of chemotherapy (5florouracil or its derivatives; S-1; tegafur; folinic acid; irinotecan; cis-platinum; gemcitabine). The aim was to evaluate safety and possible benefits of lentinan, especially on tolerance to chemotherapy and quality of life. Patients were tested also for the rate of fluorescein-labeled lentinan binding to CD14+ peripheral blood monocytes evaluated by flow cytometry. The results indicated a better tolerance to chemotherapy and an improved score of the quality of life test in the patients treated for 12 weeks with lentinan. The relationship between level of improvement and positivity of peripheral blood monocytes to lentinan binding was significant $(p<0.05)$, confirming the possible role of this test to identify the best responders to lentinan treatment, as with gastric cancer patients.

\section{Hepatocellular Carcinoma}

The Hepatocellular Carcinoma (HCC) is largely associated with cirrhosis and infection by hepatitis $\mathrm{B}$ and $\mathrm{C}$ viruses and has an increasing incidence rate. $\mathrm{HCC}$ is the third leading cause of death for cancer worldwide and the fifth and seventh most frequent cancer in men and women, respectively (International Agency for Research on Cancer: http://www-dep.iarc.fr/). Its correlation with conditions inducing chronic inflammation (viral infection, cirrhosis) can offer opportunities for immunotherapeutic interventions. Various therapeutic strategies are available and a multidisciplinary approach is the most used at present including combinations of surgery, thermoablation techniques, chemoembolization, systemic chemotherapy and monoclonal antibodies (Pascual et al., 2016). The availability and diffusion of vaccination for hepatitis $\mathrm{B}$ as well as treatments for hepatitis $\mathrm{C}$ are promising for a preventive action against the HCC onset. Nevertheless, it is still necessary to improve the effectiveness of the available therapeutic protocols. The effect of lentinan on HCC was evaluated in two experimental studies: The first, conducted in vitro and in vivo using HepG2 human cell line in vitro and $\mathrm{H} 22$ cells in a syngeneic mouse model in vivo, respectively, (Zhang et al., 2016) evaluated the cellular effects of chemotherapy + lentinan and the protective effects on tumor-bearing animals. The second, used lentinan to stimulate tumor antigen expression and as an adjuvant for possible immunization against the H22 cells (Wang et al., 2015). In both experimental sets, lentinan was associated with oxiplatin chemotherapy. The in vitro study showed that the apoptosis of HepG2 cancer cells induced by oxiplatin was increased by the addition of lentinan. The synergistic effect was secondary to a downregulation of NF- $\kappa B$, Stat 3 and surviving signaling. When the H22 tumor-bearing mice were treated with the chemotherapy + lentinan combination, reduction of side effects of chemotherapy treatment were noted as well as increase of tumor control (Wang et al., 2015). Interestingly, the incubation of $\mathrm{H} 22$ cells with lentinan induced tumor cells to over express tumor antigens. Stimulated H22 cell were used to immunize the mice. The immunization was based on the use of complete cells associated with lentinan (HCL) or of antigenic material obtained from the supernatant of H22-treated cell by precipitation with ammonium-sulphate of three fractions, at $30 \%(\mathrm{Z1})$, $60 \%(\mathrm{Z} 2)$ and $80 \%(\mathrm{Z3})$ saturation. After three weekly administration of the vaccine, mice were challenged with the $\mathrm{H} 22$ cells by intraperitoneal administration. HCL and 
$\mathrm{Z} 1$ showed to induce protective effects with $100 \%$ of survival in the $\mathrm{Zl}$ and $60 \%$ survival in the HCL immunized groups. The immunizing antigen was identified as a59.6 kDa protein. Splenocyte proliferation was increased in the Z1 and HCL groups as well as the spleen did not reduce its volume in comparison with the untreated animal model $(p<0.01)$ (Wang et al., 2015). This data suggest lentinan not only to exert an immunomodulatory action on DCs, but also a direct effect as an enhancer of tumor cell immunogenicity and as a vaccine adjuvant.

Two clinical papers reported the use of lentinan in HCC patients. Isoda et al. (2009) evaluated the effects of superfine dispersed lentinan as a food supplement administered to 36 patients with unresectable or recurrent HCC. The period of supplementation varied from 7 to over 47 weeks and significantly longer survival was observed in patients who were able to receive the treatment for a period longer than 7-12 weeks $(p<0.05)$. This was in accordance to that seen in gastric cancer patients. Other studies (Hazama et al., 2009; Yoshino et al., 2010a), confirmed the correlation between the response to lentinan treatment and the rate of lentinan binding to CD14+ peripheral blood monocytes. Lentinan-high-binding patients survived significantly longer than lentinan-low-binding patients with a median survival time of 16.3 months Vs 12.5 months, respectively $(p<0.05)$ (Isoda et al., 2009). Another study compared different therapeutic approaches to treat HCC in 78 patients with multifocal tumors (mean diameter, $6.5 \pm 0.7 \mathrm{~cm})$ : Transcatheter Arterial Chemoembolization (TACE) alone; multi-electrode Radiofrequencies Ablation (RFA) alone; the combination of RFA and TACE; and the combination of RFA, TACE and lentinan (Yang et al., 2008). Comparing the TACE and RFA alone, the first was less active than RFA in producing necrosis $(37.5 \% \mathrm{Vs}$ $47.8 \%$ ) and in reducing the percentage of tumor recurrence $(45.8 \%$ Vs $34.7 \%)$. The combination of TACE + RFA ameliorated both the results to $60.3 \%$ and $29.0 \%$ for necrosis and recurrence rate, respectively. These last results compared with the association of lentinan with TACE+RFA showed a significant $(p<0.05)$ improvement with the additional of lentinan; with necrosis reaching the $88.6 \%$ and the percentage of recurrence reduced to $17.8 \%$. The survival in the tripleassociation group was also increased, reaching a mean of 28.2 months. The bulk of these studies suggest a direct effect of lentinan on HCC that can be synergic with the lentinan-induced immunomodulation.

\section{Lung Cancer}

Lentinan capability as a therapeutic enhancer and/or immunomodulator for lung cancer was evaluated in one in vitro study, using the A549 NSCLC cells (Liu et al.,
2015) and in three clinical studies on NSCLC in advanced stages (III-IV) (Liu et al., 2015; Wang et al., 2006; Yin et al., 2015). The in vitro study investigated if the association of lentinan with the chemotherapeutic drug paclitaxel was beneficial. Paclitaxel is a plant alkaloid from Taxus brevifolia which produces alteration of the normal breakdown of microtubules during cell replication and cytotoxic effect on various epithelial cancers (Ojima et al., 2016). Viability of the cancer cells using either paclitaxel or lentinan as a single agent was dependent from dose (from 0.5 to $100 \mu \mathrm{g} \mathrm{mL}^{-1}$ ) and time of incubation (from 24 to $72 \mathrm{~h}$ ). Both treatments showed similar trend of progressive cytotoxic effect with the increasing of dose and time. While after $72 \mathrm{~h}$ the cell survival was reduced of $36.4 \%$ by paclitaxel and $47.7 \%$ by lentinan, the association of the two agents increased the effect to $72 \%$. It was also found that the paclitaxel and lentinan association increased 2.2-fold the Reactive Oxygen Species (ROS) production by A549 cells, with related $M A P 3 K 5$ and $M A P K 14$ increase and inhibitory effect on cell proliferation. Furthermore, the $72 \mathrm{~h}$ treatment demonstrated enhanced interaction of Thioredoxin-Interacting Protein (TXNIP) with NLRP3 inflammasome, followed by an increase of cleaved $C A S P 1$ and $I L 1 B$, as final events of the inflammasome activation. The process also involved induction of CASP3 activation with increased apoptosis. In conclusion, lentinan enhanced the anticancer potency of the chemotherapy by directly inducing reduction of the proliferation and increase of apoptosis in cancer cells.

Possible therapeutic efficacy of lentinan in the treatment of patients with NSCLC was evaluated (Li et al., 2011; Wang et al., 2006). The first study (Wang et al., 2006) included 81 patients (stage III-IV) who were subsequently subdivided into two groups, treated with chemotherapy (39 patients) and treated with chemotherapy + lentinan (42 patients). Follow up of Karnofsky score, body weight, lymphocyte subpopulations and incidence of chemotherapy side effects was performed. Increased $\mathrm{T}$ lymphocytes (total $\mathrm{CD} 3+$ and $\mathrm{CD} 4+$, but not $\mathrm{CD} 8+$ ) and NK cells during the chemotherapy + lentinan treatment $(p<0.01)$ suggested a cooperation between innate and adaptive immunity, with $\mathrm{T}$ helper activation. Side effects from chemotherapy were lessened in the lentinan group $(p<0.05)$ and the quality of life score was also significantly better $(p<0.01)$. The improvement of general conditions following lentinan was reported to enhance the response to chemotherapy $(50 \%$ chemotherapy + lentinan Vs 35\% chemotherapy alone). The second study (Li et al., 2011) reported a different therapeutic approach to treat NSCLC complicated by malignant effusion (cancerous hydrothorax). Sixty patient were randomly divided in two groups of 30 patients each and they were given intrathoracic injection of lentinan alone or followed by locally induced thoracic 
hyperthermia by microwave (thermotherapy). Lentinan was able to reduce the hydrothorax in both groups but with higher percentage in the double treated patients $(73.3 \%$ Vs $83 \% ; p<0.05)$. In the combination group, stability of the body weight and higher Karnofsky parameters were also observed. Similarly, blood tests confirmed that increase of CD3+, CD4+ and NK cells occurred. In both groups not particular toxicity developed and the incidence of side effects was similar in both groups $(p>0.05)$. A large number of NSCLC patients (950 from 12 studies published in China) was evaluated in a meta-analysis (Yin et al., 2015). Divided into two groups, 458 patients were treated with chemotherapy alone (control group) and 492 patient received chemotherapy + lentinan. Treatments ranged from 6 to 8 weeks (with exception of 2 weeks in only one study). Three types of chemotherapy were used: Navelbine + cisplatin (NP), gemcitabine + cisplatin (GP), or paclitaxel + cisplatin (TP). The rate of response to chemotherapy was increased by lentinan in the GP and TP groups, but not in the NP. Patients treated with chemotherapy + lentinan injection showed a reduced incidence of grades 3-4 gastrointestinal reaction and granulocytopenia. The authors concluded that lentinan had positive effects when associated with chemotherapy, but also noted the necessity of additional larger and more homogeneous studies than the ones analyzed in the meta-analysis.

\section{Intraperitoneal and Intrathoracic Malignant Effusions}

Hydrothorax and ascites can be the consequence of pleural and peritoneal cancer diffusion, creating distress to the patients as well as worsening their quality of life and prognosis. Two clinical studies on small groups of patients tested the combination of lentinan with OK432, a biological preparation consisting in a lyophilized mixture of group A Streptococcus pyogenes indicated to elicit and sustain Th1 response in cancer patients (Rebuffini et al., 2012; Saito et al., 1984). Yoshino treated 20 patients reporting $81 \%$ of response, 10 complete responses and 7 partial responses, after 1-3 intracavitary treatments. Quality of life was improved and toxicity from the treatment negligible, with low grade fever (approximately $37^{\circ} \mathrm{C}$ ) as the most common effect (Yoshino et al., 2010b). The same protocol of treatment was followed by Kawaoka on a group of 10 patients (Kawaoka et al., 2005). A total of 8 patients showed complete or partial remission. The levels of IL-12 and IFN $\gamma$ cytokines, detected in the ascites due to gastric cancer spread, was significantly increased after the second treatment than after the first treatment, while IL-10 was only slightly reduced (Kawaoka et al., 2005). Despite the limited number of observations, these studies confirmed the advantage of treatments that include lentinan.

\section{Sarcoma}

Six papers reported the results of lentinan anticancer properties on in vitro and in vivo sarcoma models. The in vitro tests were performed using the mouse sarcoma-180 (S-180) cell line. These studies investigated the cytotoxicity of various lentinan forms: With various content of Man and Gal; in triple-helix conformation or extracted as a single flexible chain; sulphated or not sulphated; with or without bound proteins (Jeff et al., 2013; Wang and Zhang, 2009; Zhang et al., 2005; Zheng et al., 2015). The common conclusion was that the level of cytotoxicity and inhibition of proliferation was almost none in one chain and highly purified form of lentinan and that the forms with bound proteins were more active. The best activities were developed by triple chain form, followed by the sulphate derivatives. The molecular weight was affecting the functionality of the derivative. A dose-dependent effectiveness was described (Jeff et al., 2013). In two studies, the HT-29 and HCT-116 human colon cancer cell lines were also challenged with lentinan. The adherent epithelial cancer cells were less sensible to the treatment than the nonadherent S-180 in both studies (Jeff et al., 2013; Zheng et al., 2015). In vivo models were used to test a mannogalactoglucan (LE-MGG) and various fractions of water-extracted lentinan with different molecular weight (Zheng et al., 2015). Tumors were obtained by in-mouse implantation of S-180, HT-29 and HCT-116 cells. The LE-MGG produced tumor growth inhibition in a dosedependent manner. Within the different molecular weight derivatives, the triple-helix lentinan with molecular weight $1.49 \times 10^{6}$ inhibited the tumor growth in a proportion of $49.5 \%$, approximating the inhibitory effect of 5-fluorouracil $(50.5 \%)$. The use of single chain preparations produced, instead, negligible response. Two other in vivo studies used different sarcoma models. One study used implanted S908D2 sarcoma cells in B10.D2 mice to compare the effect of lentinan Vs micellapist, another $\beta$-glucan obtained from $L$. edodes mushroom (Maruyama et al., 2006). While micellapist was clearly not active, lentinan was able to significantly reduce the tumor growth by eliciting a cytotoxic antitumor reaction. The test on splenocytes from tumor-bearing mice treated with lentinan showed that the reaction was mediated by CD8+ cells and CD4+ cells and inhibited by the specific antibodies. The second study, instead, used Balb/c mice inoculated on both flanks with Meth A fibrosarcoma cells but with different molecular weights: $1 \times 10^{6}$ on the right side and $2 \times 10^{5}$ on the left side ("double grafted tumor system") (Ebina, 2005; Kohya et al., 1989). Betaglucans prepared from Coliolus versicolor, Tricholoma matsutake, Agaricus blazei and L. edodes were compared by intratumoral administration in the right flank tumor. The lentinan was the only purified $\beta$-glucan without bound proteins. Antitumor activities resulted for 
the various product, but lentinan was without effects on both primary and metastatic tumor (Ebina, 2005). However, it is interesting to note that in a previous study (Suga et al., 1984) testing different strains of mice (DBA/2, SWM/Ms, A/J, C3H/He, C57BL/6 and Balb/c mice), low or no effects of lentinan treatment was found in $\mathrm{C} 3 \mathrm{H} / \mathrm{He}, \mathrm{C} 57 \mathrm{BL} / 6$ and $\mathrm{Balb} / \mathrm{c}$ mice; this is contrary to the positive responses with the other strains. The choice of the model might be critical for addressing correct evaluation of the response to treatments with lentinan (and, possibly, other $\beta$-glucans) in experimental studies.

\section{Other Cancers}

A selection of other cancers was only occasionally evaluated for possible beneficial effects of lentinan administration. One clinical study of esophageal carcinoma, evaluated 50 patients to compare the treatment with tegafur Vs tegafur with lentinan (Wang et al., 2012). Increased serum levels of proinflammatory and Th1 response cytokines (IL-2, IL-6 and IL-12) during the treatments, as well as decrease of the inhibitory and Th2 cytokines (IL-4, IL-5 and IL-10), were significantly present in patients of both groups $(p<0.05)$. These changes were more evident in the lentinan-association group than in the control group $(p<0.05)$. The Karnofsky and ECOG scores were better in both groups after two cycles of treatment, but with more improvement in quality of life and performance status after the lentinan treatment $(p<0.05)$.

A study of 29 pancreatic cancer patients with unresectable or recurrent neoplasm were treated with superfine dispersed lentinan (Shimizu et al., 2009) and another 10 patients received the superfine dispersed lentinan in another study (Yagi et al., 2010), in association with S-1. Both studies reported an improvement of quality of life, minimal side effects due to lentinan (grade 1 diarrhea) and improved survival rate.

The T24 human urothelial (bladder) cancer cell line was tested in two studies (Bao et al., 2015; Sun et al., 2015). Both used various concentrations of lentinan, alone or associated to gemcitabine. Apoptosis and necrosis induced by the treatment were verified by flow cytometry. Mitochondrial membrane potential of T24 cells and intracellular ROS were also evaluated at the confocal microscope by intracellular fluorescence staining with JC-1 and DCFH-DA probes. Inhibition of cancer cell proliferation and viability was dose related and appeared secondary to mitochondrial depolarization and ROS generation possibly via activation of no mitochondrial pathway.

The protective effect of lentinan was also evidenced in acute myeloid leukemia treated with idarubicin and cytarabine, with or without lentinan supplementation, in a Brown Norway rat model. The lentinan reduced body gain and cachexia, increased cytotoxic T lymphocytes and downregulated IL-4, IL10 and IL-6 (McCormack et al., 2010).

Finally, three experimental studies were developed,in vitro and in vivo, to investigate effects of lentinan on squamous cell cancers and melanoma. The laryngeal squamous cancer cell line Hep-2 was used to test a new derivative from $L$. edodes presenting mannose, arabinose, galactose, xylose and rhamnose in its composition. Formation of colonies, proliferation and invasion were reduced by high concentration of the lentinan derivative (Cao et al., 2013). Human oral squamous cell carcinoma was implanted in nude mice and treated with S-1 with lentinan administered intraperitoneally. The tumor response was evaluated for apoptosis using TUNEL assay and for variation in expression of Thymidylate Synthase (TYMS), Dihydropyrimidine Dehydrogenase (DPYD) and Orotate Phosphoribosyl Transferase (OPRT) genes by RTPCR and ELISA. Apoptosis was enhanced by the addition of lentinan to therapy. In addition, downregulation of TYMS and DPYD mRNA and upregulation of OPRT mRNA were induced with supposed effects on cell proliferation. General conditions of the lentinan-treated animals were better than in control, with no loss of body weight (Harada et al., 2010). The classic mouse melanoma $\mathrm{B} 16 \mathrm{~F} 10$ model was produced in C57BL/6 mice and used by Wang and collaborators for testing the efficacy of a DC vaccine based on gp100 antigen (Wang et al., 2007). The lentinan was used either alone or in combination with gp100-DC. The vaccine was effective with enhancement of the induced immunological effects (increased cytotoxic $\mathrm{T}$ lymphocytes and NK cells associated with increased IL-2 and IFN $\gamma$ in the splenocytes) by lentinan. The association of lentinan with the DC vaccine inhibited the tumor growth, increased the immune cell infiltrate inside and peripherally to the tumor and determined a $66.7 \%$ of survival in the treated animals. The results indicated a synergistic effect of lentinan with the DC vaccine (Wang et al., 2007).

\section{Conclusion}

The effects of lentinan and of its possible derivatives can be summarized as follows: Direct cytotoxic activity on tumor cells of various types in vitro, inducing apoptosis by mechanisms involving telomerase and inflammasome regulation; shift from $\mathrm{Th} 2$ to $\mathrm{Th} 1$ response, enhancing the anticancer response and supporting DC vaccines; general amelioration of the conditions of the treated individuals in both in vivo and in vitro studies; in the clinical studies, the improvement of performance status was associated in some cases to increased survival and this was related to the percentage of CD14+ cells able to bind lentinan; the oral treatment with the superfine dispersed lentinan was giving positive results as the parenteral administration; good palliative effect and low side effects were the most common 
conclusion of the studies. Most studies, with the exception of one (Yoshino et al., 2016), presented results that were concordant on one main point of interest, that is the advantage to introduce lentinan supplement in the treatment of cancer patients in association to the appropriate chemotherapy. Interestingly, the increased performance status and the reduction of the chemotherapy side effects by lentinan association allowed prolonged treatment to patients, favoring their survival. It might be possible that the better-responding patients already had traits or characteristics for a higher tolerance to the therapy. However, two meta-analyses confirmed the advantage of lentinan use (Oba et al., 2009; Yin et al., 2015). Larger clinical studies, not only from the Asiatic countries, should be performed to confirm the described beneficial effects of lentinan. The problem must be addressed first, the availability of standard product of lentinan - with clearly known composition and quality - to permit full evaluation and replication of the results. Finally, the use of the mushroom derivatives should be controlled for quality and possible contaminations, other factors that could influence the effectiveness and safeness of the tested products (Money, 2016).

\section{Acknowledgement}

We thank the support of institutional grants RVO 61388971 (CZ), UniCredit Bank (CZ) and ARPA Foundation, Pisa (IT).

\section{Author's Contributions}

Dr. Luca Vannucci, MD, PhD. was responsible of the paper ideation, organization and writing; Dr. Petr Šíma, PhD. participated with data collection and critical discussion; Prof. Vaclav Vetvicka, PhD. cured the critical revision and editing; Dr. Jiří Křižan, PhD followed the paper collection and preparation of tables.

\section{Ethics}

The Authors declare no conflicts of interest.

\section{References}

Bao, L., Y. Wang, R. Ma, X. Ren and R. Cheng et al., 2015. Apoptosis-inducing effects of lentinan on the proliferation of human bladder cancer T24 cells. Pak. J. Pharm. Sci., 28: 1595-1600. PMID: 26408880

Cao, X., R. Liu, J. Liu, Y. Huo and W. Yang et al., 2013. A novel polysaccharide from Lentinus edodes Mycelia exhibits potential antitumor activity on laryngeal squamous cancer cell line Hep-2. Applied Biochem. Biotechnol., 171: 1444-1453.

DOI: $10.1007 / \mathrm{s} 12010-013-0441-6$
Chihara, G., 1983. Preclinical evaluation of lentinan in animal models. Adv. Exp. Med. Biol., 166: 189-197. DOI: $10.1007 / 978-1-4757-1410-4 \_16$

Chihara, G., J. Hamuro, Y.Y. Maeda, T. Shiio and T. Suga et al., 1987. Antitumor and metastasisinhibitory activities of lentinan as an immunomodulator: An overview. Cancer Detect. Prev. Suppl., 1: 423-43. PMID: 3319150

Ebina, T., 2005. [Antitumor effects of intratumoral injection of Basidiomycetes preparations]. Gan Kagaku Ryoho, 32: 1654-1656. PMID: 16315899

Ferlay, J., I. Soerjomataram, R. Dikshit, S. Eser and C. Mathers et al., 2015. Cancer incidence and mortality worldwide: Sources, methods and major patterns in GLOBOCAN 2012. Int. J. Cancer, 136: E359-E386. DOI: 10.1002/ijc.29210

Goodridge, H.S., C.N. Reyes, C.A. Becker, T.R. Katsumoto and J. Ma et al., 2011. Activation of the innate immune receptor Dectin-1 upon formation of a 'phagocytic synapse'. Nature, 472: 471-475. DOI: 10.1038/nature10071

Harada, K., Y. Itashiki, T. Takenawa and Y. Ueyama, 2010. Effects of lentinan alone and in combination with fluoropyrimidine anticancer agent on growth of human oral squamous cell carcinoma in vitro and in vivo. Int. J. Oncol., 37: 623-631.

DOI: 10.3892/ijo_00000711

Hazama, S., S. Watanabe, M. Ohashi, M. Yagi and M. Suzuki et al., 2009. Efficacy of orally administered superfine dispersed lentinan (beta-1,3-glucan) for the treatment of advanced colorectal cancer. Anticancer Res., 29: 2611-267. PMID: 19596936

Higashi, D., K. Seki, Y. Ishibashi, Y. Egawa and M. Koga et al., 2012. The effect of lentinan combination therapy for unresectable advanced gastric cancer. Anticancer Res. 32: 2365-8. PMID: 22641676

Hori, T., T. Ikehara, S. Takatsuka, T. Fukuoka and M. Tendo et al., 2011. [Combination chemotherapy of S-1/low-dose CDDP/lentinan for advanced gastric cancer]. Gan. Kagaku Ryoho, 38: 293-295. PMID: 21368498

Ina, K., R. Furuta, T. Kataoka, S. Kayukawa and T. Yoshida et al., 2011. Lentinan prolonged survival in patients with gastric cancer receiving S-1-based chemotherapy. World J. Clin. Oncol., 2: 339-343. DOI: $10.5306 /$ wjco.v2.i10.339

Ina, K., T. Kataoka and T. Ando, 2013. The use of lentinan for treating gastric cancer. Anticancer Agents Med. Chem., 13: 681-688. DOI: $10.2174 / 1871520611313050002$

Isoda, N., Y. Eguchi, H. Nukaya, K. Hosho and Y. Suga et al., 2009. Clinical efficacy of superfine dispersed lentinan (beta-1,3-glucan) in patients with hepatocellular carcinoma. Hepatogastroenterology, 56: 437-441. PMID: 19579616 
Jeff, I.B., S. Li, X. Peng, R.M. Kassim and B. Liu et al., 2013. Purification, structural elucidation and antitumor activity of a novel mannogalactoglucan from the fruiting bodies of Lentinus edodes. Fitoterapia, 84: 338-346. DOI: 10.1016/j.fitote.2012.12.008

Kataoka, H., T. Shimura, T. Mizoshita, E. Kubota and Y. Mori et al., 2009. Lentinan with S-1 and paclitaxel for gastric cancer chemotherapy improve patient quality of life. Hepatogastroenterology, 56: 547-550. PMID: 19579640

Kawaoka, T., S. Yoshino, H. Kondo, K. Yamamoto and S. Hazama et al., 2005. [Clinical evaluation of intrapleural or peritoneal repetitive administration of Lentinan and OK-432 for malignant effusion]. Gan Kagaku Ryoho, 32: 1565-7. PMID: 16315870

Kohya, H., T. Ebina, T. Yamaguchi and N. Ishida, 1989. The "double grafted tumor system", proposed to find effector cells in the analyses of antitumor effect of BRMs. Biotherapy, 1: 139-151.

DOI: $10.1007 / \mathrm{bf0} 2170883$

Leibundgut-Landmann, S., F. Osorio, G.D. Brown and C. Reis e Sousa, 2008. Stimulation of dendritic cells via the dectin-1/Syk pathway allows priming of cytotoxic T-cell responses. Blood, 112: 4971-4980. DOI: 10.1182/blood-2008-05-158469

Li, X.J., Y.J. Jia and L. Chen, 2011. [Clinical observation of thermotherapy combined with thoracic injection of lentinan in treatment of cancerous hydrothorax of patients with lung cancer]. Zhongguo Zhong Xi Yi Jie He Za Zhi, 31: 1062-1065. PMID: 21910335

Liu, W., J. Gu, J. Qi, X.N. Zeng and J. Ji et al., 2015. Lentinan exerts synergistic apoptotic effects with paclitaxel in A549 cells via activating ROS-TXNIPNLRP3 inflammasome. J. Cell Mol. Med., 19: 1949-1955. DOI: $10.1111 / \mathrm{jcmm} .12570$

Maruyama, S., Y. Sukekawa, Y. Kaneko and S. Fujimoto, 2006. [Anti tumor activities of lentinan and micellapist in tumor-bearing mice]. Gan Kagaku Ryoho, 33: 1726-179. PMID: 17212088

McCormack, E., J. Skavland, M. Mujic, O. Bruserud and B.T. Gjertsen, 2010. Lentinan: hematopoietic, immunological and efficacy studies in a syngeneic model of acute myeloid leukemia. Nutr. Cancer, 62: 574-583. DOI: 10.1080/01635580903532416

Molitoris, H.P., 1994. Mushrooms in medicine. Folia Microbiol., 39: 91-98. DOI: 10.1007/bf02906801

Money, N.P., 2016. Are mushrooms medicinal? Fungal Biol., $\quad 120$ : 449-453. DOI: 10.1016/j.funbio.2016.01.006

Mushiake, H., T. Tsunoda, M. Nukatsuka, K. Shimao and M. Fukushima et al., 2005. Dendritic cells might be one of key factors for eliciting antitumor effect by chemoimmunotherapy in vivo. Cancer Immunol. Immunother., 54: 120-128.

DOI: $10.1007 / \mathrm{s} 00262-004-0585-\mathrm{x}$
Nimura, H., N. Mitsumori, N. Takahashi, H. Kashimura and S. Takayama et al., 2006. [S-1 combined with lentinan in patients with unresectable or recurrent gastric cancer]. Gan Kagaku Ryoho, 33 Suppl 1: 106-109. PMID: 16897983

Novak, M. and V. Vetvicka, 2009. Glucans as biological response modifiers. Endocr. Metab. Immune Disord. Drug. Targets, 9: 67-75. DOI: $10.2174 / 187153009787582423$

Oba, K., M. Kobayashi, T. Matsui, Y. Kodera and J. Sakamoto, 2009. Individual patient based metaanalysis of lentinan for unresectable/recurrent gastric cancer. Anticancer Res. 29: 2739-45. PMID: 19596954

Ojima, I., B. Lichtenthal, S. Lee, C. Wang and X. Wang, 2016. Taxane anticancer agents: A patent perspective. Expert Opin. Ther. Pat., 26: 1-20. DOI: $10.1517 / 13543776.2016 .1111872$

Oka, M., S. Hazama, M. Suzuki, F. Wang and K. Wadamori et al., 1996. In vitro and in vivo analysis of human leukocyte binding by the antitumor polysaccharide, lentinan. Int. J. Immunopharmacol., 18: 211-216. DOI: 10.1016/0192-0561(95)00115-8

Parveen, A., B. Parveen, R. Parveen and S. Ahmad, 2015. Challenges and guidelines for clinical trial of herbal drugs. J. Pharm. Bioallied. Sci., 7: 329-333. DOI: $10.4103 / 0975-7406.168035$

Pascual, S., I. Herrera and J. Irurzun, 2016. New advances in hepatocellular carcinoma. World J. Hepatol., 8: 421-438. DOI: 10.4254/wjh.v8.i9.421

Rebuffini, E., L. Zuccarino, E. Grecchi, F. Carinci and V.E. Merulla, 2012. Picibanil (OK-432) in the treatment of head and neck lymphangiomas in children. Dent. Res. J., 9: S192-S196. DOI: $10.4103 / 1735-3327.109752$

Saito, M., M. Nanjo, E. Aonuma, T. Noda and I. Nakadate et al., 1984. Activated macrophages are responsible for the tumor-inhibitory effect in mice receiving intravenous injection of OK-432. Int. J. Cancer, 33: 271-276. DOI: 10.1002/ijc.2910330217

Schreibelt, G., K.F. Bol, E.H. Aarntzen, W.R. Gerritsen and C.J. Punt et al., 2013. Importance of helper T-cell activation in dendritic cell-based anticancer immunotherapy. Oncoimmunology, 2: e24440-e24440. DOI: 10.4161 /onci.24440

Shimizu, K., S. Watanabe, S. Watanabe, K. Matsuda and T. Suga et al., 2009. Efficacy of oral administered superfine dispersed lentinan for advanced pancreatic cancer. Hepatogastroenterology, 56: 240-244. PMID: 19453066

Shirasaka, T., Y. Shimamato, H. Ohshimo, M. Yamaguchi and T. Kato et al., 1996. Development of a novel form of an oral 5-fluorouracil derivative (S-1) directed to the potentiation of the tumor selective cytotoxicity of 5-fluorouracil by two biochemical modulators. Anticancer Drugs., 7: 548-557. DOI: $10.1097 / 00001813-199607000-00010$ 
Sreenivasulu, K., M. Vijayalakshmi and K.R. Sambasivarao, 2010. Regulation studies of telomerase gene in cancer cells by lentinan. Avicenna J. Med. Biotechnol., 2: 181-185. PMID: 23408759

Suga, T., T. Shiio, Y.Y. Maeda and G. Chihara, 1984. Antitumor activity of lentinan in murine syngeneic and autochthonous hosts and its suppressive effect on 3-methylcholanthrene-induced carcinogenesis. Cancer Res., 44: 5132-5137. PMID: 6488173

Sun, M., W. Zhao, Q. Xie, Y. Zhan and B. Wu, 2015. Lentinan reduces tumor progression by enhancing gemcitabine chemotherapy in urothelial bladder cancer. Surg. Oncol., 24: 28-34. DOI: $10.1016 /$ j.suronc.2014.11.002

Tzianabos, A.O., 2000. Polysaccharide immunomodulators as therapeutic agents: Structural aspects and biologic function. Clin. Microbiol. Rev., 13: 523-533. DOI: $10.1128 / \mathrm{cmr}$.13.4.523-533.2000

Vannucci, L., J. Krizan, P. Sima, D. Stakheev and F. Caja et al., 2013. Immunostimulatory properties and antitumor activities of glucans (Review). Int. J. Oncol., 43: 357-364. DOI: 10.3892/ijo.2013.1974

Wang, J., Z.D. Zhou and D.J. Xia, 2007. [Study on effect of lentinan in enhancing anti-tumor action of dendritic cytoma vaccine and its mechanism]. Zhongguo Zhong Xi Yi Jie He Za Zhi, 27: 60-64. PMID: 17302067

Wang, J.L., Z. Bi, J.W. Zou and X.M. Gu, 2012. Combination therapy with lentinan improves outcomes in patients with esophageal carcinoma. Mol. Med. Rep., 5: 745-748. DOI: 10.3892/mmr.2011.718

Wang, W., X. Dai and X. Ouyang, 2006. [Efficacy of Lentinan combined with chemotherapy in advanced non-small cell lung cancer]. Zhongguo Fei Ai Za Zhi, 9: 78-81. DOI: 10.3779/j.issn.1009-3419.2006.01.20

Wang, X. and L. Zhang, 2009. Physicochemical properties and antitumor activities for sulfated derivatives of lentinan. Carbohydr Res. 344: 2209-2216. DOI:10.1016/j.carres.2009.04.033

Wang, Y., X. Han, Y.D. Li, S.Y. Zhao and D.J. Zhang et al., 2015. Effects of tumor-specific antigen induced by lentinan on murine $\mathrm{H} 22$ hepatocellular carcinoma immunoprophylaxis. Eur. Rev. Med. Pharmacol. Sci., 19: 4516-4524. PMID: 26698247

Wasser, S.P., 2002. Medicinal mushrooms as a source of antitumor and immunomodulating polysaccharides. Applied Microbiol. Biotechnol., 60: 258-274. DOI: $10.1007 / \mathrm{s} 00253-002-1076-7$

Xia, Y., V. Vetvicka, J. Yan, M. Hanikyrova and T. Mayadas et al., 1999. The beta-glucan-binding lectin site of mouse CR3 (CD11b/CD18) and its function in generating a primed state of the receptor that mediates cytotoxic activation in response to iC3b-opsonized target cells. J. Immunol., 162: 2281-2290. PMID: 9973505
Yagi, M., S. Watanabe, S. Yoshino, S. Hazama and T. Suga et al., 2010. [Provision for adverse effect of S-1 containing chemotherapy in patients with advanced digestive cancer--combination with superfine dispersed lentinan]. Gan Kagaku Ryoho, 37: 457-462. PMID: 20332683

Yang, P., M. Liang, Y. Zhang and B. Shen, 2008. Clinical application of a combination therapy of lentinan, multi-electrode RFA and TACE in HCC. Adv. Ther., 25: 787-794. DOI: $10.1007 / \mathrm{s} 12325-008-0079-\mathrm{x}$

Yin, X., J. Ying, L. Li, H. Zhang and H. Wang, 2015. A meta-analysis of lentinan injection combined with chemotherapy in the treatment of nonsmall cell lung cancer. Indian J. Cancer, 52: e29-31. DOI: 10.4103/0019-509X.168953

Yoshino, S., K. Nishikawa, S. Morita, T. Takahashi and K. Sakata et al., 2016. Randomised phase III study of S-1 alone versus S-1 plus lentinan for unresectable or recurrent gastric cancer (JFMC360701). Eur. J. Cancer, 65: 164-171.

DOI: $10.1016 /$ j.ejca.2016.06.012

Yoshino, S., S. Watanabe, M. Imano, T. Suga and S. Nakazawa et al., 2010a. Improvement of QOL and prognosis by treatment of superfine dispersed lentinan in patients with advanced gastric cancer. Hepatogastroenterology, 57: 172-7. PMID: 20422897

Yoshino, S., S. Yoshida, N. Maeda, Y. Maeda and K. Maeda et al., 2010b. [Clinical evaluation of the combination treatment of intrapleural or intraperitoneal administration of lentinan and OK432 for malignant effusion]. Gan Kagaku Ryoho, 37: 2798-2800. PMID: 21224717

Zhang, L., X. Li, X. Xu and F. Zeng, 2005. Correlation between antitumor activity, molecular weight and conformation of lentinan. Carbohydr. Res., 340: 1515-1521. DOI: 10.1016/j.carres.2005.02.032

Zhang, Y., Q. Li, J. Wang, F. Cheng and X. Huang et al., 2016. Polysaccharide from Lentinus edodes combined with oxaliplatin possesses the synergy and attenuation effect in hepatocellular carcinoma. Cancer Lett., 377: 117-125.

DOI: $10.1016 /$ j.canlet.2016.04.037

Zhao, L., Y. Xiao and N. Xiao, 2013. Effect of lentinan combined with docetaxel and cisplatin on the proliferation and apoptosis of BGC823 cells. Tumour. Biol., 34: 1531-1536. DOI: $10.1007 / \mathrm{s} 13277-013-0680-8$

Zheng, Y., M. Hao, H. Nan, I. Jeff and Y. Zhou et al., 2015. Relationship of chemical composition and cytotoxicity of water-soluble polysaccharides from Lentinus edodes fruiting bodies. Pak. J. Pharm. Sci., 28: 1069-1074. PMID: 26051725 